Article

\title{
Total and Double Total Domination Number on Hexagonal Grid
}

\author{
Antoaneta Klobučar ${ }^{1, *}$ and Ana Klobučar ${ }^{2}$ \\ 1 Faculty of Economics, University of Osijek, 31000 Osijek, Croatia \\ 2 Faculty of Mechanical Engineering and Naval Architecture, University of Zagreb, 10000 Zagreb, Croatia; \\ aklobucar@fsb.hr \\ * Correspondence: aneta@efos.hr
}

Received: 25 October 2019; Accepted: 13 November 2019; Published: 15 November 2019

\begin{abstract}
In this paper, we determine the upper and lower bound for the total domination number and exact values and the upper bound for the double-total domination number on hexagonal grid $H_{m, n}$ with $m$ hexagons in a row and $n$ hexagons in a column. Further, we explore the ratio between the total domination number and the number of vertices of $H_{m, n}$ when $m$ and $n$ tend to infinity.
\end{abstract}

Keywords: total domination number; double-total domination number; hexagonal grid; molecular graph

\section{Introduction}

Graph dominations are widely applied in different problems such as dominating queens, computer network, school bus routing, and social network problems. Specifically, graph dominations have huge applications in chemistry [1-5]. Chemical structures can be represented by graphs, where vertices and edges represent atoms and chemical bonds, respectively. Because of such a correspondence, many chemical and physical properties of molecules are in correlation with graph theoretical invariants. One very important such invariant is the total (double) domination number [2,6-10].

In this paper, we consider hexagonal grids with $m$ hexagons in a row and $n$ hexagons in a column and also infinite hexagonal grids. Hexagonal systems are geometric objects that are obtained by arranging congruent regular hexagons in a plane. They are of significant importance in theoretical chemistry as a natural graph representation of benzenoid hydrocarbons $[1,5,11]$. Benzenoid hydrocarbons and their derivates are an important class of organic compounds, which have, apart from their chemical importance, great technical and pharmaceutical importance as well and belong to the class of the most serious polluters of the environment.

We explore total and double-total dominations on an arbitrary hexagonal grid. We give upper and lower bound for the totally dominating number and the upper bound for the double-totally dominating number. Furthermore, we explore the ratio between the total domination number and the number of vertices of $H_{m, n}$ when $m$ and $n$ tend to infinity. At this moment, there are only few publications on total and double-total domination on hexagonal chains [3,12], but none dealing with arbitrary grids.

Apart from this Introduction, the rest of the paper is organized as follows. Section 2 lists preliminaries about total and double domination, dominating sets, and hexagonal systems. Section 3 gives upper and lower bounds for total domination number $\gamma_{t}$ on arbitrary hexagonal grid $H_{m, n}$. Further, Section 4 is concerned with the ratio between the total domination number and the number vertices of $H_{m, n}$ when $m$ and $n$ tend to infinity. Section 5 deals with double-total domination and gives double-total domination number $\gamma \times 2 t$ for linear hexagonal chain $H_{m, 1}$ and the upper bound for arbitrary hexagonal grid $H_{m, n}$. The final Section 6 gives conclusions and future work. 


\section{Preliminaries}

Let $G$ be a graph with the vertex set $V(G)$ and edge set $E(G)$. A set $D \subset V(G)$ we call a dominating set of a graph $G$ if every vertex $y$ in $V(G) \backslash D$ is adjacent to some vertex in $D$. Domination number $\gamma(G)$ is the cardinality of the smallest dominating set. Total domination is the stronger version of domination. A set $D \subset V(G)$ is a totally dominating set of a graph $G$ if every vertex $y$ in $V(G)$ is adjacent to some vertex in $D$. The total domination number $\gamma_{t}(G)$ is the cardinality of the smallest totally dominating set.

A set $S \subseteq V$ is a $k$-tuple dominating set if every vertex $v \in V \backslash S$ satisfies $\operatorname{deg}_{S}(v) \geq k$ (vertex $v$ is adjacent to at least $k$ vertices from the set $S$ ). The $k$-tuple domination number is the minimum cardinality among all $k$-tuple dominating sets. A set $S \subseteq V$ is a $k$-tuple totally dominating set ( $k$-totally dominating set) if every vertex $v \in V$ satisfies $\operatorname{deg}_{S}(v) \geq k$, e.g., each vertex in $V$ has at least $k$ neighbors in $S$. In such a case, it must be $k \leq \delta$ where $\delta$ is the minimum degree of vertices on $G$ and $|S| \geq k+1$. The $k$ - tuple total domination number $\gamma_{\times k t}(G)\left(k\right.$-total domination number $\left.\gamma_{k t}(G)\right)$ is the cardinality of the smallest $k$-tuple totally dominating set. For $k=2$, the two-tuple totally dominating set is called the double-totally dominating set.

Each vertex in a hexagonal system has either degree two or degree three. It follows that on the hexagonal grid, there is no $\times k$-total domination for $k \geq 3$.

A vertex shared by three hexagons is called an internal vertex of the respective hexagonal system. A hexagonal system where no three hexagons have an intersection (no internal vertices) is called a catacondensed system, else it is pericondensed.

A catacondensed hexagonal system in which every hexagon is adjacent to at most two hexagons is called a hexagonal chain. A linear hexagonal chain is a hexagonal chain that is a graph representation of linear polyacene. The linear hexagonal chain with $m$ hexagons will be denoted by $H(m, 1)$. A double hexagonal chain consists of two condensed identical hexagonal chains $(H(m, 2))$.

\section{Total Domination Number of a Hexagonal Grid with $m$ Hexagons in a Row and $n$ Hexagons in a Column}

We denote by $H_{m, n}$ a hexagonal grid with $m$ hexagons in a row and $n$ hexagons in a column. For $n=1$, we have a linear hexagonal chain, and for $n=2$, a double hexagonal chains. In $H_{m, n}$, any zigzag line with no vertical edges is called a horizontal zigzag line. The horizontal zigzag line of $H_{m, n}$ is denoted by $L_{i},(1 \leq i \leq n+1)$, where vertices on $L_{i}$ are $v_{i, 1}, v_{i, 2}, \ldots, v_{i, 2 m}, v_{i, 2 m+1}$, if $i \in\{1, n+1\}$. Otherwise, for each $L_{i}, 2 \leq i \leq n$, we have vertices $v_{i, 1}, v_{i, 2}, \ldots, v_{i, 2 m+2}$. Therefore, on $H_{m, n}$, there are $2(2 m+1)+(n-1) \cdot(2 m+2)=2(m n+m+n)$ vertices. See Figure 1 for an example for $H_{4,3}$.

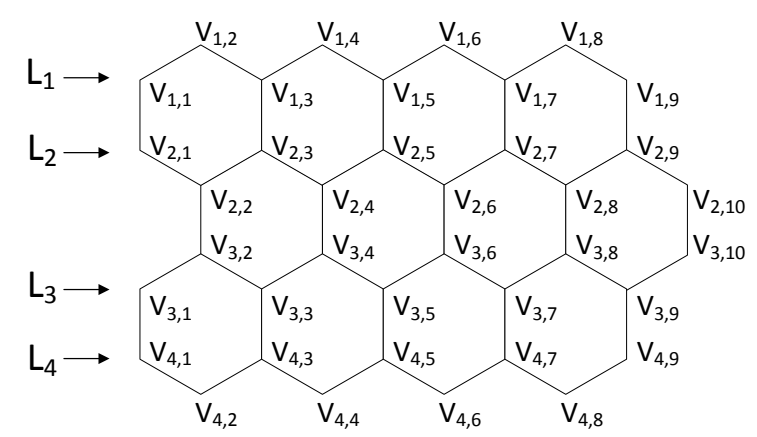

Figure 1. Horizontal zigzag lines denoted by $L_{i}, i=1, \ldots 4$, and vertices of $H_{4,3}$. Grid $H_{4,3}$ has $m=4$ hexagons in a row and $n=3$ hexagons in a column. As an example, $L_{1}$ consists of vertices $v_{1,1}, v_{1,2}$, $v_{1,3}, v_{1,4}, v_{1,5}, v_{1,6}, v_{1,7}, v_{1,8}, v_{1,9}$. Similarly, $L_{2}$ consists of vertices $v_{2,1}, v_{2,2}, v_{2,3}, v_{2,4}, v_{2,5}, v_{2,6}, v_{2,7}, v_{2,8}$, $v_{2,9}, v_{2,10}$. 
It is known [9] that for the cycle $C_{n}$, it holds that:

$$
\gamma_{t}\left(C_{n}\right)=\left\{\begin{array}{cl}
\frac{n}{2}+1, & n \equiv 2(\bmod 4) \\
\left\lceil\frac{n}{2}\right\rceil, & \text { otherwise }
\end{array}\right.
$$

For linear and double hexagonal chains, the following two theorems are proven $[3,4]$ :

Theorem 1. ([4]) For a linear hexagonal chain with $m$ hexagons, it holds that:

$$
\gamma_{t}\left(H_{m, 1}\right)=2 m+2
$$

Theorem 2. ([3]) For a double hexagonal chain with $m$ hexagons, it holds that:

$$
\gamma_{t}\left(H_{m, 2}\right) \leq\left\{\begin{array}{cc}
\frac{5 m}{2}+2, & m \equiv 0(\bmod 4) \\
10\left\lfloor\frac{m}{4}\right\rfloor+6, & m \equiv 1(\bmod 4) \\
10\left\lfloor\frac{m}{4}\right\rfloor+8, & m \equiv 2(\bmod 4) \\
10\left\lceil\frac{m}{4}\right\rceil, & m \equiv 3(\bmod 4)
\end{array}\right.
$$

Now, we will consider $H_{m, n}$ when $n \geq 3$ and give the upper bounds for total domination number $\gamma_{t}\left(H_{m, n}\right)$.

Theorem 3. For a hexagonal grid with $m$ hexagons in a row and $n$ hexagons $(n \geq 3)$ in a column $H_{m, n}$, it holds that:

$$
\gamma_{t}\left(H_{m, n}\right) \leq\left\{\begin{array}{cc}
(n+2) \frac{2 m+3}{3}-1, & m \equiv 0(\bmod 3) \\
(n+2) \frac{2 m+4}{3}-2, & m \equiv 1(\bmod 3) \\
(n+2) \frac{2 m+2}{3}, & m \equiv 2(\bmod 3)
\end{array}\right.
$$

Proof. We will consider six different cases depending on $m$ modulo three and $n$ modulo two $(n$ is odd or even).

Case $1.1 m \equiv 0(\bmod 3), n$ odd.

Let us define $S_{i}=\left\{v_{i, 2+3 j}, j=0, \ldots, \frac{2 m}{3}\right\}, i=2, \ldots, n, S_{1}=\left\{v_{1,5+6 j}, j=0, \ldots, \frac{m}{3}-1\right\}$ and $S_{n+1}=\left\{v_{n+1,5+6 j}, j=0, \ldots, \frac{m}{3}-1\right\}$. See Figure 2 for an example for $H_{9,7}$.

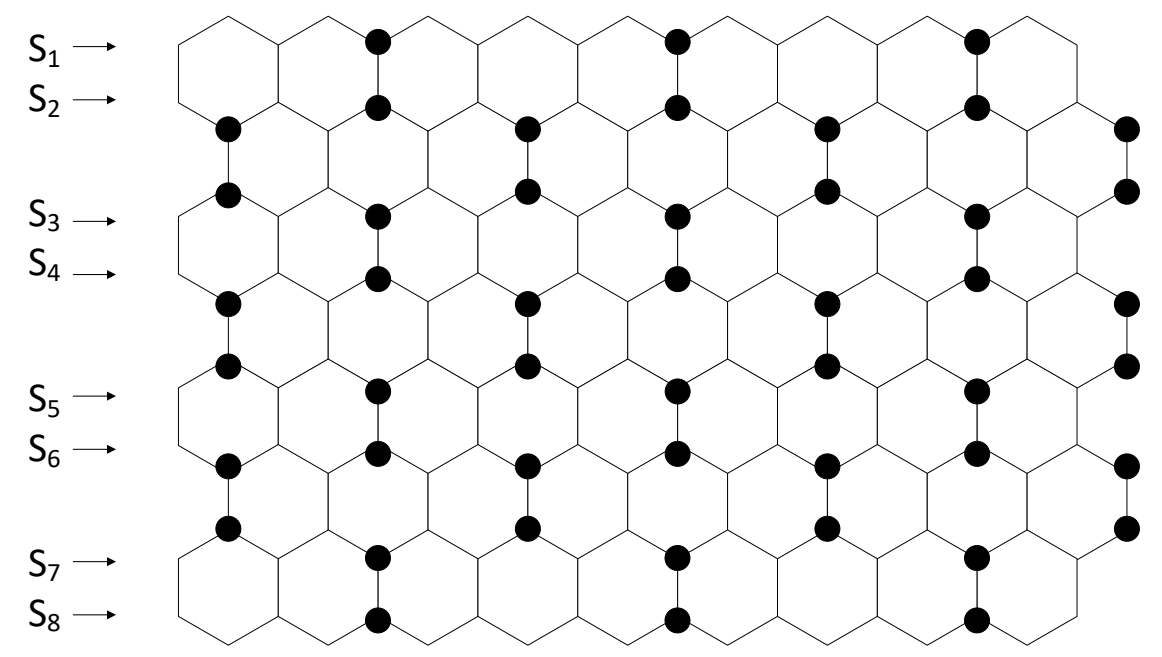

Figure 2. $S_{1} \cup S_{2} \cup \cdots \cup S_{8}$ on $H_{9,7}$. 


$$
\left|S_{1} \cup S_{2} \cup \cdots \cup S_{n+1}\right|=(n-1)\left|S_{2}\right|+2\left|S_{1}\right|=(n-1)\left(\frac{2 m}{3}+1\right)+2 \cdot \frac{m}{3}=n\left(\frac{2 m+3}{3}\right)-1 .
$$

These vertices totally dominate all vertices on $L_{2}, \ldots, L_{n}$ and also totally dominate both $3\left(\frac{m}{3}\right)=m$ vertices on the $L_{1}$ and on $L_{n+1}$.

All vertices are totally dominated by exactly one vertex. Further, all totally dominating vertices totally dominate three vertices except for totally dominating vertices from the last column. The totally dominating vertices from the last column have degree two, so they can totally dominate two vertices at most.

Now, we consider the case on $L_{1}$ (it is the same for $L_{n+1}$ ). From the previous, $m$ vertices on $L_{1}$ are totally dominated, and $m+1$ are not totally dominated. From the structure of $S_{1}$, it follows that there exist $\frac{m}{3}$ groups of three consequent vertices, which are not totally dominated. From each such group, we must take two vertices into the totally dominating set $D$. It follows that we have to take $2 \cdot \frac{m}{3}$ such vertices to dominate $L_{1}$ totally. Then, only $v_{1,2 m+1}$ is not totally dominated $\left(v_{n+1,2 m+1}\right.$ for $\left.L_{n+1}\right)$, so we need at least one vertex more to dominate it totally. Finally,

$$
\gamma_{t}\left(H_{m, n}\right) \leq n\left(\frac{2 m+3}{3}\right)-1+4 \frac{m}{3}+2=(n+2) \frac{2 m}{3}+(n+1)=(n+2) \frac{2 m+3}{3}-1 .
$$

Case $1.2 m \equiv 0(\bmod 3), n$ even.

In this case, $S_{1}, S_{2}, \ldots, S_{n}$ are the same as in Case 1.1. Only $S_{n+1}=\left\{v_{n+1,1+6 j}, j=0, \ldots, \frac{m}{3}\right\}$. Then, $\left|S_{n+1}\right|=\frac{m}{3}+1$. Therefore,

$$
\begin{aligned}
\left|S_{1} \cup S_{2} \cup \cdots \cup S_{n+1}\right| & =(n-1)\left|S_{2}\right|+2\left|S_{1}\right|+1=(n-1)\left(\frac{2 m}{3}+1\right)+2 \cdot \frac{m}{3}+1 \\
& =n \cdot\left(\frac{2 m}{3}+1\right)=n\left(\frac{2 m+3}{3}\right) .
\end{aligned}
$$

On $L_{1}$ and $L_{n+1}$ (same for odd $n$ ), we need $2 \cdot \frac{2 m}{3}=\frac{4 m}{3}$ totally dominating vertices. In this case, only the vertex $v_{1,2 m+1}$ is not totally dominated, and we need at least one vertex more to dominate it totally. From this follows:

$$
\gamma_{t}\left(H_{m, n}\right) \leq n \cdot\left(\frac{2 m}{3}+1\right)+4 \cdot \frac{m}{3}+1=(n+2) \frac{2 m}{3}+(n+1)=(n+2) \frac{2 m+3}{3}-1 .
$$

Case $2.1 m \equiv 1(\bmod 3), n$ odd.

Let us define $S_{i}=\left\{v_{i, 2+3 j}, j=0, \ldots,\left\lfloor\frac{2 m}{3}\right\rfloor\right\}, i=2, \ldots, n, S_{1}=\left\{v_{1,5+6 j}, j=0, \ldots,\left\lfloor\frac{m}{3}\right\rfloor-1\right\}$, and $S_{n+1}=\left\{v_{n+1,5+6 j}, j=0, \ldots,\left\lfloor\frac{m}{3}\right\rfloor-1\right\}$. See Figure 3 for an example for $H_{10,7}$.

$$
\left|S_{1} \cup S_{2} \cup \cdots \cup S_{n+1}\right|=(n-1)\left|S_{2}\right|+2\left|S_{1}\right|=(n-1)\left(\left\lfloor\frac{2 m}{3}\right\rfloor+1\right)+2 \cdot\left\lfloor\frac{m}{3}\right\rfloor .
$$

These vertices totally dominate all vertices on $L_{2}, \ldots, L_{n}$ except for the last column. Furthermore, they totally dominate $3\left(\left\lfloor\frac{m}{3}\right\rfloor\right)$ vertices on $L_{1}$ and the same on $L_{n+1}$.

All vertices in this case are totally dominated by exactly one vertex, and each totally dominating vertex totally dominates three vertices (which is maximum on a hexagon).

Now, we consider the case on $L_{1}$ (it is the same on $L_{n+1}$ ). On $L_{1}$ exist $\left\lceil\frac{m}{3}\right\rceil$ groups of three consequent vertices, which are not totally dominated. From each such group, we have to take at least two vertices into the totally dominating set $D$. It follows that we have to take $2 \cdot\left\lceil\frac{m}{3}\right\rceil$ such vertices to dominate $L_{1}$ totally. 


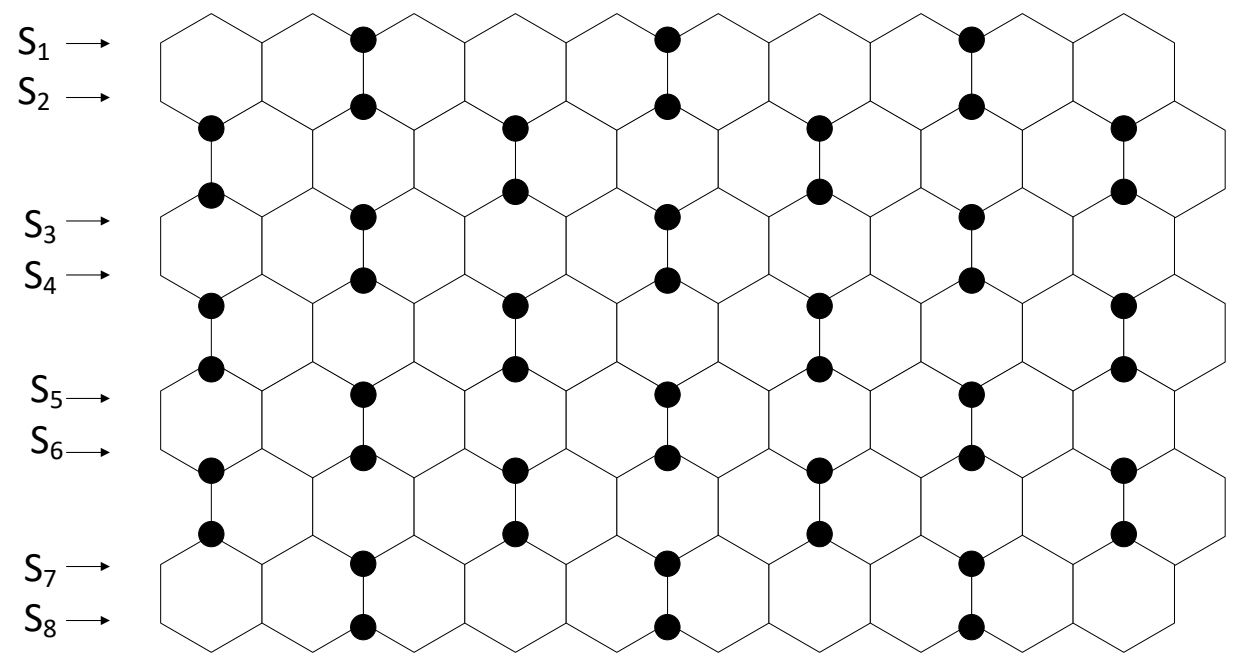

Figure 3. $S_{1} \cup S_{2} \cup \cdots \cup S_{8}$ on $H_{10,7}$.

Then, on the last $n^{\text {th }}$ column, $2 \cdot\left\lfloor\frac{n}{2}\right\rfloor$ vertices are not totally dominated. They are in groups of two consequent vertices:

$$
\left(v_{2,2 m+2}, v_{3,2 m+2} ; v_{4,2 m+2}, v_{5,2 m+2} ; \ldots ; v_{n-1,2 m+2} v_{n, 2 m+2}\right) .
$$

See Figure 3 for an example for $H_{10,7}$. Therefore, to dominate them totally, we must take all of them in the totally dominating set $D$. Hence,

$$
\begin{aligned}
\gamma_{t}\left(H_{m, n}\right) & \leq(n-1)\left(\left\lfloor\frac{2 m}{3}\right\rfloor+1\right)+2 \cdot\left\lfloor\frac{m}{3}\right\rfloor+4 \cdot\left\lceil\frac{m}{3}\right\rceil+2 \cdot\left\lfloor\frac{n}{2}\right\rfloor \\
& =\frac{2}{3}(m n+2 m+2 n+1)=(n+2) \frac{2 m+1}{3}+n=(n+2) \frac{2 m+4}{3}-2 .
\end{aligned}
$$

Case $2.2 m \equiv 1(\bmod 3), n$ even.

In this case, $S_{1}, S_{2}, \ldots, S_{n}$ are the same as in Case 2.1 for odd $n$. Only $S_{n+1}=\left\{v_{n+1,1+6 j}, j=\right.$ $\left.0, \ldots,\left\lfloor\frac{m}{3}\right\rfloor\right\}$. Then, $\left|S_{n+1}\right|=\left\lfloor\frac{m}{3}\right\rfloor+1$. Therefore,

$$
\begin{aligned}
\left|S_{1} \cup S_{2} \cup \cdots \cup S_{n+1}\right| & =(n-1)\left|S_{2}\right|+2\left|S_{1}\right|+1 \\
& =(n-1)\left(\left\lfloor\frac{2 m}{3}\right\rfloor+1\right)+2 \cdot\left\lfloor\frac{m}{3}\right\rfloor+1 .
\end{aligned}
$$

On $L_{1}$, we need $2 \cdot\left\lceil\frac{m}{3}\right\rceil$ totally dominating vertices more, and on $L_{n+1}$, we need $2 \cdot\left\lfloor\frac{m}{3}\right\rfloor$ totally dominating vertices more (there is one block more with three undominated vertices on the $L_{1}$ ).

The same as for the case when nis odd, there are $2 \cdot \frac{n}{2}$ vertices, which are not totally dominated on the last $n^{\text {th }}$ column. Furthermore, to dominate them totally, we must take all of them in the totally dominating set $D$. Hence,

$$
\begin{aligned}
\gamma_{t}\left(H_{m, n}\right) & \leq\left((n-1)\left(\left\lfloor\frac{2 m}{3}\right\rfloor+1\right)+2 \cdot\left\lfloor\frac{m}{3}\right\rfloor+1\right)+2 \cdot\left\lceil\frac{m}{3}\right\rceil+2 \cdot\left\lfloor\frac{m}{3}\right\rfloor+2 \cdot \frac{n}{2} \\
& =\frac{2}{3}(m n+2 m+2 n+1)=(n+2) \frac{2 m+4}{3}-2 .
\end{aligned}
$$

Case $3.1 m \equiv 2(\bmod 3), n$ odd. 
Similar to the previous cases, $S_{i}=\left\{v_{i, 2+3 j}, j=0, \ldots,\left\lfloor\frac{2 m}{3}\right\rfloor\right\}, i=2, \ldots, n, S_{1}=\left\{v_{1,5+6 j}, j=\right.$ $\left.0, \ldots,\left\lfloor\frac{m}{3}\right\rfloor\right\}$, and $S_{n+1}=\left\{v_{n+1,5+6 j}, j=0, \ldots,\left\lfloor\frac{m}{3}\right\rfloor\right\}$. See Figure 4 for an example for $H_{8,7}$.

$$
\begin{aligned}
\left|S_{1} \cup S_{2} \cup \cdots \cup S_{n+1}\right| & =(n-1)\left|S_{2}\right|+2\left|S_{1}\right| \\
& =(n-1)\left(\left\lfloor\frac{2 m}{3}\right\rfloor+1\right)+2 \cdot\left(\left\lfloor\frac{m}{3}\right\rfloor+1\right)=n \frac{2 m+2}{3} .
\end{aligned}
$$

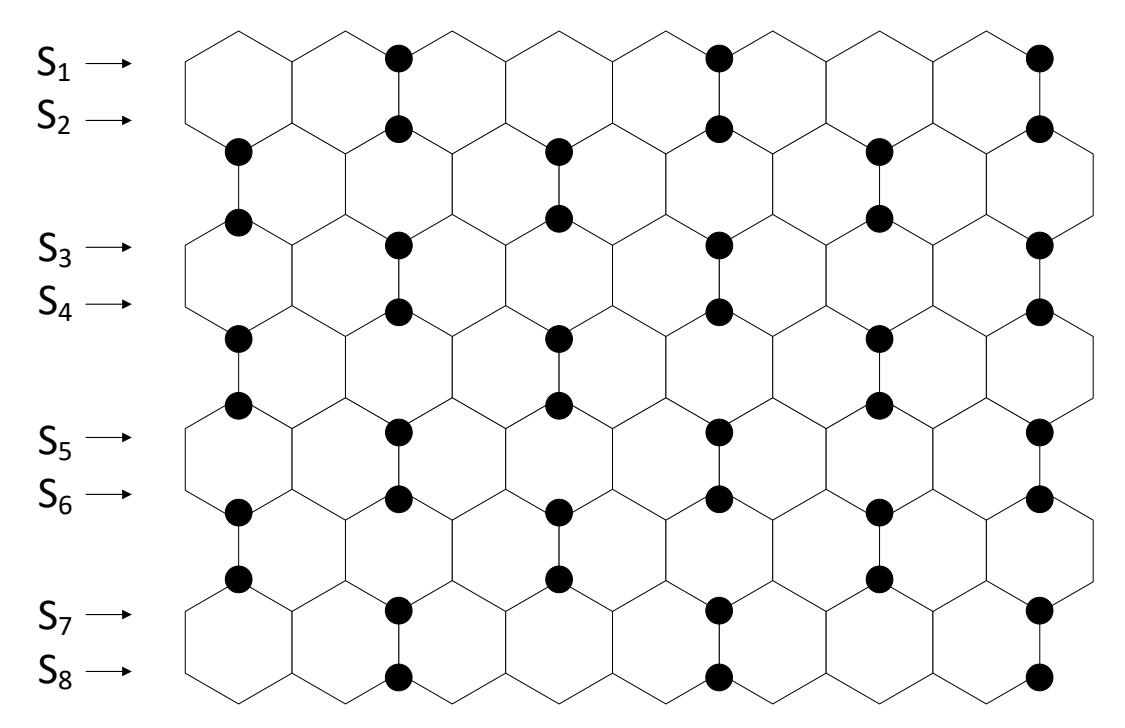

Figure 4. $S_{1} \cup S_{2} \cup \cdots \cup S_{8}$ on $H_{8,7}$.

These vertices totally dominate all vertices on $L_{2}, \ldots, L_{n}$ and also totally dominate $3\left\lfloor\frac{m}{3}\right\rfloor+2$ vertices both on $L_{1}$ and $L_{n+1}$.

All vertices are totally dominated by exactly one vertex. Further, all totally dominating vertices totally dominate three vertices except for $v_{1,2 m+1}$ and $v_{n+1,2 m+1}$, which totally dominate two vertices. Vertices $v_{1,2 m+1}$ and $v_{n+1,2 m+1}$ have degree two, so they can totally dominate two vertices at most.

Now, we consider the case on $L_{1}$ (it is the same on $\left.L_{n+1}\right)$. Similar to the case $m \equiv 1(\bmod 3)$, on $L_{1}$ exist $\left\lceil\frac{m}{3}\right\rceil$ groups of three consequent vertices, which are not totally dominated. From each such group, we have to take at least two vertices into the totally dominating set $D$. It follows that we have to take $2 \cdot\left\lceil\frac{m}{3}\right\rceil$ such vertices to dominate $L_{1}$ totally. Then, all vertices on $H_{m, n}$ are total dominated.

$$
\begin{aligned}
\gamma_{t}\left(H_{m, n}\right) & \leq n \frac{2 m+2}{3}+4 \cdot\left\lceil\frac{m}{3}\right\rceil \\
& =n \frac{2 m+2}{3}+4 \cdot \frac{m+1}{3}=(n+2) \frac{2 m+2}{3}
\end{aligned}
$$

Case $3.2 m \equiv 2(\bmod 3), n$ even.

In this case, $S_{1}, S_{2}, \ldots, S_{n}$ are the same as in Case 3.1. Only $S_{n+1}=\left\{v_{n+1,1+6 j}, j=0, \ldots,\left\lfloor\frac{m}{3}\right\rfloor\right\}$. Then, $\left|S_{n+1}\right|=\left\lfloor\frac{m}{3}\right\rfloor+1$. These vertices totally dominate all vertices on $L_{2}, \ldots L_{n}$, and each vertex is totally dominated by exactly one vertex.

$$
\left|S_{1} \cup S_{2} \cup \cdots \cup S_{n+1}\right|=(n-1)\left|S_{2}\right|+2\left|S_{1}\right|=(n-1)\left(\left\lfloor\frac{2 m}{3}\right\rfloor+1\right)+2 \cdot\left(\left\lfloor\frac{m}{3}\right\rfloor+1\right) .
$$

The same as in Case 3.1, we need 2 $\left\lceil\frac{m}{3}\right\rceil$ vertices to dominate $L_{1}$ totally (also for $L_{n+1}$ ). From this, it follows:

$$
\gamma_{t}\left(H_{m, n}\right) \leq(n-1)\left(\left\lfloor\frac{2 m}{3}\right\rfloor+1\right)+6 \cdot\left\lceil\frac{m}{3}\right\rceil=(n+2) \frac{2 m+2}{3} .
$$


Remark 1. It is easy to check that the bounds are tight for small examples. For example, it holds that $\gamma_{t}\left(H_{2,3}\right)=10, \gamma_{t}\left(H_{2,4}\right)=12, \gamma_{t}\left(H_{3,3}\right)=14$, and $\gamma_{t}\left(H_{4,3}\right)=18$. All these numbers are equal to the upper bound for $\gamma_{t}\left(H_{m, n}\right)$. See Figure 5 for an example for $H_{3,3}$.

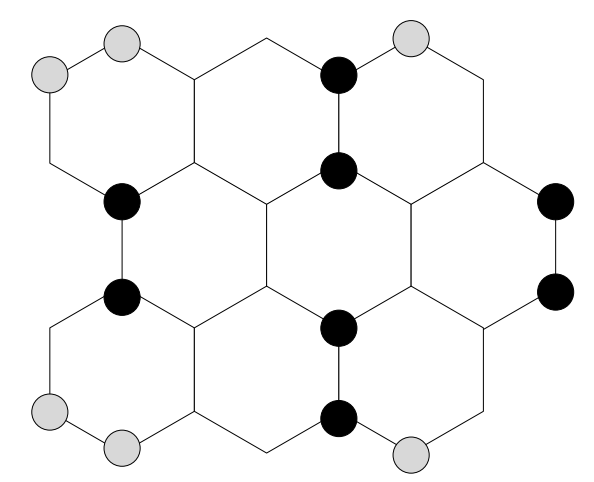

Figure 5. Total dominating set on $H_{3,3}$. Black vertices belong to $S_{1} \cup S_{2} \cup \cdots \cup S_{4}$. Gray vertices must be added to the totally dominating set to dominate $L_{1}, L_{4}$ and the last column $n=3$.

Proposition 1. For a hexagonal grid with $m$ hexagons in a row and $n$ hexagons $(n \geq 3)$ in a column $H_{m, n}$, it holds that:

$$
\gamma_{t}\left(H_{m, n}\right)>\frac{2 n m}{3} .
$$

Proof. It follows from Theorem 3 if we take only $S_{1} \cup \cdots \cup S_{n+1}$ as a part of the totally dominating set on $H_{m, n}$. Vertices on $H_{m, n}$ are then totally dominated with only one vertex from $S_{1} \cup \cdots \cup S_{n+1}$ or are not totally dominated. Then:

$$
\gamma_{t}\left(H_{m, n}\right)>\left|S_{1} \cup \cdots \cup S_{n+1}\right|>\frac{2 n m}{3} .
$$

4. Determining $\lim _{m, n \rightarrow \infty} \frac{\gamma_{t}\left(H_{m, n}\right)}{2(m n+m+n)}$

Theorem 4. For a hexagonal grid with $m$ hexagons in a row and $n$ hexagons in a column $H_{m, n}$, which has $2(m n+m+n)$ vertices, it holds that:

$$
\lim _{m, n \rightarrow \infty} \frac{\gamma_{t}\left(H_{m, n}\right)}{2(m n+m+n)}=\frac{1}{3} .
$$

Proof. From Proposition 1 and Theorem 3, it follows that:

$$
\frac{2 n m}{3}<\gamma_{t}\left(H_{m, n}\right)<\frac{2(n+2)(m+2)}{3} .
$$

If we divide this by $2(m n+m+n)=2((m+1)(n+1)-1)$ (the number of vertices on $\left.H_{m, n}\right)$, we obtain:

$$
\begin{gathered}
\frac{2 n m}{3 \cdot 2((m+1)(n+1)-1)}<\frac{\gamma_{t}\left(H_{m, n}\right)}{2((m+1)(n+1)-1)} \\
<\frac{2(n+2)(m+2)}{3 \cdot 2((m+1)(n+1)-1)} .
\end{gathered}
$$


For $m, n \rightarrow \infty$, the left and the right hand side of (1) tend to $\frac{1}{3}$. Applying the sandwich rule gives us the desired result:

$$
\lim _{m, n \rightarrow \infty} \frac{\gamma_{t}\left(H_{m, n}\right)}{2(m n+m+n)}=\frac{1}{3}
$$

The previous limit is no surprise because on a hexagonal grid, one vertex can totally dominate at most three vertices. This means that on very large grids, around one third of their vertices should be in any totally dominating set.

\section{Double-Total Domination on a Hexagonal Grid}

Theorem 5. For a linear hexagonal chain with $m$ hexagons, it holds that:

$$
\gamma_{\times 2 t}\left(H_{m, 1}\right)=\left\{\begin{aligned}
6\left\lceil\frac{m}{2}\right\rceil, & \text { modd } \\
6 \frac{m}{2}+4, & \text { m even } .
\end{aligned}\right.
$$

Proof. (a) $m$ is odd.

Because we consider double-total domination, each vertex adjacent to a vertex with degree two must be in any minimal double-totally dominating set $D$. From this, it follows that all vertices from the first and last hexagon and all other vertices with degree three must be in $D$. If only these vertices are in $D$, inner vertices of degree three are not $\times 2$-totally dominated. They are totally dominated only once. We have to take at least two vertices of degree two on each odd hexagon to double-totally dominate them. Then, $\left\{\left(v_{i, 1+4 j}\right),\left(v_{i, 2+4 j}\right),\left(v_{i, 3+4 j}\right) ; i \in\{1,2\} ; j \in\left\{0,1, \ldots,\left\lfloor\frac{m}{2}\right\rfloor\right\}\right\}$ are in $D$. Hence,

$$
|D|=6\left(\left\lfloor\frac{m}{2}\right\rfloor+1\right)=6\left\lceil\frac{m}{2}\right\rceil .
$$

(b) $m$ is even.

Like in the previous case, any minimal double-totally dominating set must have all vertices that are adjacent to at least one vertex of degree two. If only these vertices are in $D$, the inner vertices of degree three are not double-totally dominated. Therefore, we have to take into $D$ the remaining two inner vertices from every odd hexagon. Then, $\left\{\left(v_{i, 1+4 j}\right),\left(v_{i, 2+4 j}\right),\left(v_{i, 3+4 j}\right) ; i \in\{1,2\} ; j \in\left\{0,1, \ldots, \frac{m}{2}-1\right\}\right\}$ $\cup\left\{v_{1,2 m}, v_{1,2 m+1}, v_{2,2 m}, v_{2,2 m+1}\right\}$ are in $D$. Therefore,

$$
|D|=6 \frac{m}{2}+4
$$

Theorem 6. For a hexagonal grid with $m$ hexagons in a row and $n$ hexagons in a column $H_{m, n}$, it holds that:

$$
\gamma_{\times 2 t}\left(H_{m, n}\right) \leq\left\{\begin{array}{cl}
(3 n+3)\left\lceil\frac{m}{2}\right\rceil+n-1, & \text { m, n odd } \\
n\left(\frac{3 m}{2}+2\right)+2 m+1, & \text { m, } n \text { even } \\
(n+1)\left(\frac{3 m}{2}+2\right), & \text { m even, } n \text { odd } \\
3 n\left(\frac{m+1}{2}\right)+2 m+n & \text { modd, } n \text { even }
\end{array}\right.
$$

Proof. Case 1. $m, n$ are odd.

By $T_{i}$, we denote the subset of the double-totally dominating set on the $i^{\text {th }}$ zigzag line of $H_{m, n}$. Let us define:

$$
\begin{aligned}
T_{1} & =\left\{v_{1,1+4 j}, v_{1,2+4 j}, v_{1,3+4 j}, j=0, \ldots,\left\lfloor\frac{m}{2}\right\rfloor\right\} \\
T_{i} & =\left\{v_{i, 1+4 j}, v_{i, 2+4 j}, v_{i, 3+4 j}, j=0, \ldots,\left\lfloor\frac{m}{2}\right\rfloor\right\} \cup v_{i, 2 m+2}, i=2, \ldots, n
\end{aligned}
$$




$$
T_{n+1}=\left\{v_{n+1,1+4 j}, v_{n+1,2+4 j}, v_{n+1,3+4 j}, j=0, \ldots,\left\lfloor\frac{m}{2}\right\rfloor\right\} .
$$

See Figure 6 for an example for $H_{5,5}$. It is easy to see that $T_{1} \cup T_{2} \cup \cdots \cup T_{n+1}$ is a double-totally dominating set on $H_{m, n}$ for $m$ and $n$ odd.

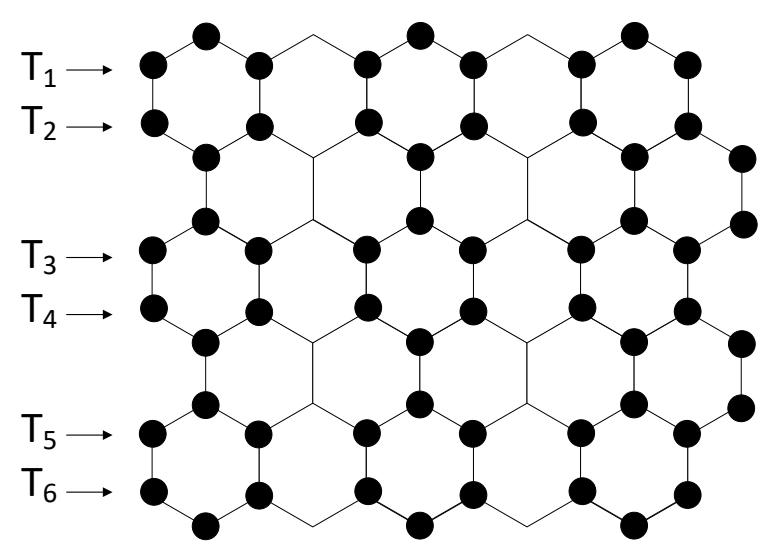

Figure 6. $T_{1} \cup T_{2} \cup \cdots \cup T_{6}$ on $H_{5,5}$.

We have $\left|T_{1} \cup T_{2} \cup \cdots \cup T_{n+1}\right|=2\left|T_{1}\right|+(n-1)\left|T_{i}\right|$. This is $2 \cdot 3\left\lceil\frac{m}{2}\right\rceil+(n-1)\left(3\left\lceil\frac{m}{2}\right\rceil+1\right)=$ $(3 n+3)\left\lceil\frac{m}{2}\right\rceil+(n-1)$. Hence,

$$
\gamma_{\times 2 t}\left(H_{m, n}\right) \leq(3 n+3)\left\lceil\frac{m}{2}\right\rceil+(n-1) .
$$

Case 2. $m, n$ are even.

Let us define:

$$
\begin{aligned}
T_{1} & =\left\{v_{1,1+4 j}, v_{1,2+4 j}, v_{1,3+4 j}, j=0, \ldots, \frac{m}{2}-1\right\} \cup\left\{v_{1,2 m}, v_{1,2 m+1}\right\} \\
T_{i} & =\left\{v_{i, 1+4 j}, v_{i, 2+4 j}, v_{i, 3+4 j}, j=0, \ldots, \frac{m}{2}-1\right\} \cup\left\{v_{i, 2 m+1}, v_{i, 2 m+2}\right\}, i=2, \ldots, n \\
T_{n+1} & =\left\{v_{n+1,1}, v_{n+1,2}, \ldots, v_{n+1,2 m+1}\right\} .
\end{aligned}
$$

See Figure 7 for an example for $H_{6,6}$. It is easy to see that $T_{1} \cup T_{2} \cup \cdots \cup T_{n+1}$ is a double-totally dominating set on $H_{m, n}$ for $m$ and $n$ even. $\left|T_{1} \cup T_{2} \cup \cdots \cup T_{n+1}\right|=n\left|T_{1}\right|+\left|T_{n+1}\right|$. This is $n\left(\frac{3 m}{2}+2\right)+$ $2 m+1$. Therefore,

$$
\gamma \times 2 t\left(H_{m, n}\right) \leq n\left(\frac{3 m}{2}+2\right)+2 m+1
$$

Case 3. $m$ is even, and $n$ is odd.

Let us define:

$$
\begin{aligned}
T_{1} & =\left\{v_{1,1+4 j}, v_{1,2+4 j}, v_{1,3+4 j}, j=0, \ldots, \frac{m}{2}-1\right\} \cup\left\{v_{1,2 m}, v_{1,2 m+1}\right\} \\
T_{i} & =\left\{v_{i, 1+4 j}, v_{i, 2+4 j}, v_{i, 3+4 j}, j=0, \ldots, \frac{m}{2}-1\right\} \cup\left\{v_{1,2 m+1}, v_{1,2 m+2}\right\}, i=2, \ldots, n \\
T_{n+1} & =\left\{v_{n+1,1+4 j}, v_{n+1,2+4 j}, v_{n+1,3+4 j}, j=0, \ldots, \frac{m}{2}-1\right\} \cup\left\{v_{n+1,2 m}, v_{n+1,2 m+1}\right\} .
\end{aligned}
$$

See Figure 8 for an example for $H_{6,5}$. It is easy to see that $T_{1} \cup T_{2} \cup \cdots \cup T_{n+1}$ is a double-totally dominating set on $H_{m, n}\left|T_{1} \cup T_{2} \cup \cdots \cup T_{n+1}\right|=(n+1)\left|T_{1}\right|$. This is $(n+1)\left(\frac{3 m}{2}+2\right)$. Hence,

$$
\gamma_{\times 2 t}\left(H_{m, n}\right) \leq(n+1)\left(\frac{3 m}{2}+2\right) .
$$




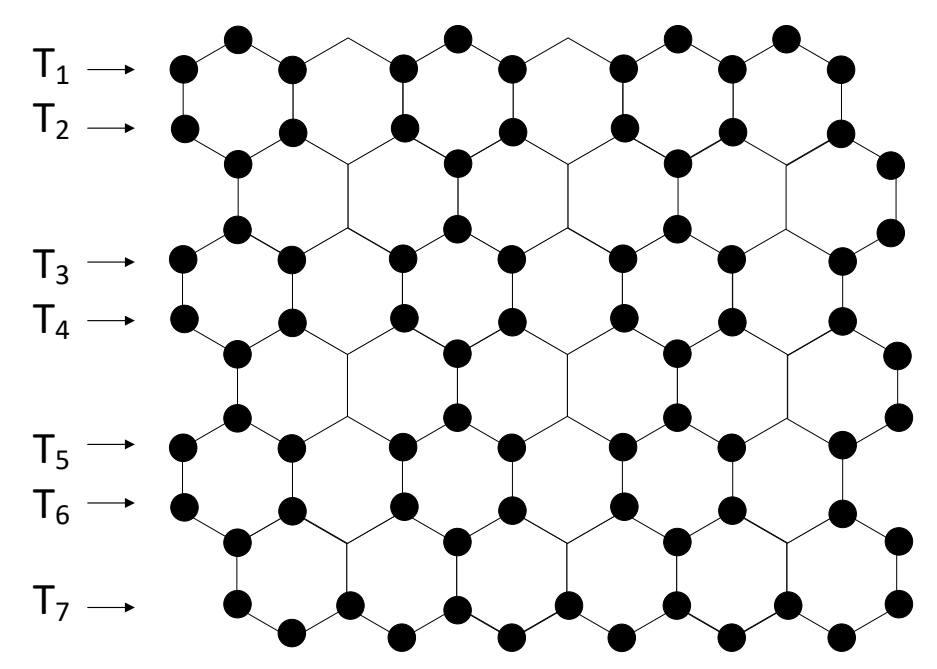

Figure 7. $T_{1} \cup T_{2} \cup \cdots \cup T_{7}$ on $H_{6,6}$.

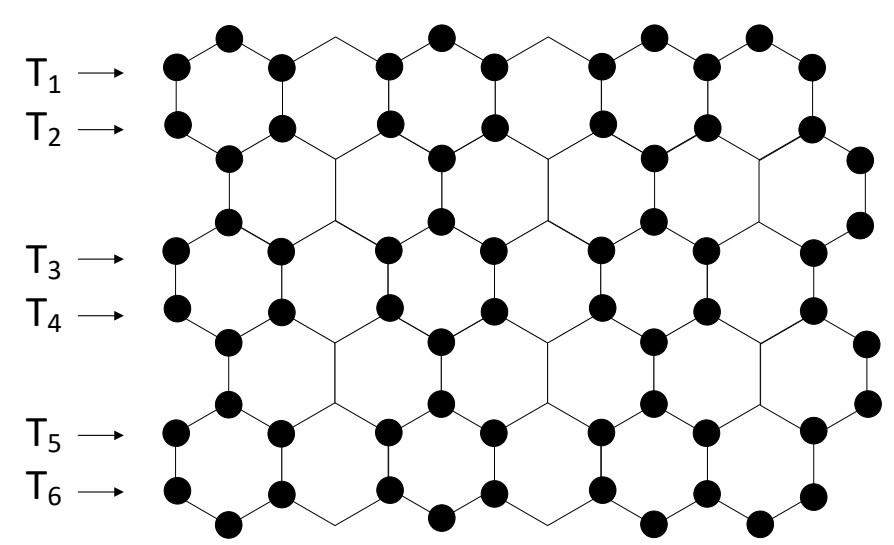

Figure 8. $T_{1} \cup T_{2} \cup \cdots \cup T_{6}$ on $H_{6,5}$.

Case 4. $m$ is odd, and $n$ is even.

Let us define:

$$
\begin{aligned}
T_{1} & =\left\{v_{1,1+4 j}, v_{1,2+4 j}, v_{1,3+4 j}, j=0, \ldots,\left\lfloor\frac{m}{2}\right\rfloor\right\} \\
T_{i} & =\left\{v_{i, 1+4 j}, v_{i, 2+4 j}, v_{i, 3+4 j}, j=0, \ldots,\left\lfloor\frac{m}{2}\right\rfloor\right\} \cup\left\{v_{1,2 m+2}\right\}, i=2, \ldots, n \\
T_{n+1} & =\left\{v_{n+1,1}, v_{n+1,2}, \ldots, v_{n+1,2 m+1}\right\} .
\end{aligned}
$$

See Figure 9 for an example for $H_{5,6}$. It is easy to see that $T_{1} \cup T_{2} \cup \cdots \cup T_{n+1}$ is a double-totally dominating set on $H_{m, n}$. Then, it holds $\left|T_{1} \cup T_{2} \cup \cdots \cup T_{n+1}\right|=\left|T_{1}\right|+(n-1)\left|T_{2}\right|+\left|T_{n+1}\right|$. This is $3\left(\left\lfloor\frac{m}{2}\right\rfloor+1\right)+(n-1)\left(3\left(\left\lfloor\frac{m}{2}\right\rfloor+1\right)+1\right)+2 m+1=3 n\left(\frac{m+1}{2}\right)+2 m+n$.

Therefore,

$$
\gamma \times 2 t\left(H_{m, n}\right) \leq 3 n\left(\frac{m+1}{2}\right)+2 m+n .
$$




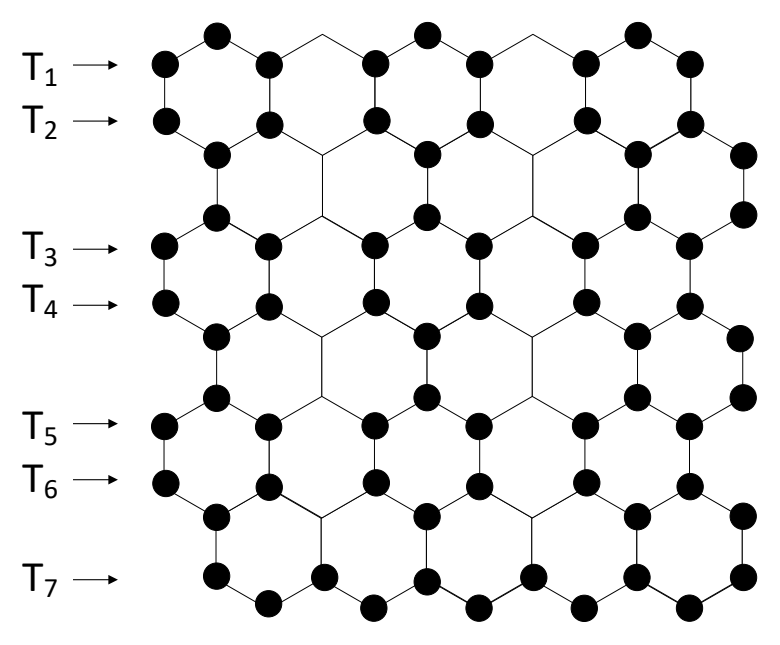

Figure 9. $T_{1} \cup T_{2} \cup \cdots \cup T_{7}$ on $H_{5,6}$.

\section{Conclusions}

We determined the upper and lower bounds for the total domination number and exact values and the upper bound for the double-total domination number on hexagonal grid $H_{m, n}$ with $m$ hexagons in a row and $n$ hexagons in a column. Previous works [3,12] explored the total domination on hexagonal chains, but none dealt with arbitrary hexagonal grids. We tried to fill this gap. Further, we showed that the ratio between the total domination number $\gamma_{t}\left(H_{m, n}\right)$ and the number of vertices of $H_{m, n}$ when $m$ and $n$ tend to infinity equals $1 / 3$. This means that for very large grids, around a third of the vertices should be in the totally dominating set. Finally, we showed with multiple examples that the given bounds were tight. Moreover, the given bounds were equal to the exact solution for the given examples. In future work, we plan to determine the exact values for $\gamma_{t}\left(H_{m, n}\right)$ and $\gamma_{\times 2 t}\left(H_{m, n}\right)$. We suspect that some of the given bounds are minimal. Furthermore, we plan to explore total and double-total dominations on some other types of chemical graphs.

Author Contributions: Conceptualization, formal analysis, supervision, writing-original draft, A.K. (Antoaneta Klobučar); Data curation, formal analysis, validation, visualization, writing-review \& editing, A.K. (Ana Klobučar). All authors contributed equally.

Funding: This research received no external funding.

Conflicts of Interest: The authors declare no conflict of interest.

\section{References}

1. Gao, Y.; Zhu, E.; Shao, Z.; Gutman, I.; Klobučar, A. Total domination and open packing in some chemical graphs. J. Math. Chem. 2018, 56, 1481-1493. [CrossRef]

2. Henning, M.A; Rautenbach, D.; Schäfer, P.M. Open packing, total domination and $P_{3}$-Radon number. Discret. Math. 2009, 313, 992-998. [CrossRef]

3. Majstorović, S.; Klobučar, A. Upper bound for total domination number on linear and double hexagonal chains. Int. J. Chem. Model. 2009, 3, 139-145.

4. Mojdeh, D.A.; Habibi, M.; Badakhshian, L. Total and connected domination in chemical graphs. Ital. J. Pure Appl. Math. 2018, 39, 393-401.

5. Vukičević, D.; Klobučar, A. K-Dominating sets on linear benzenoids and on the infinite hexagonal grid. Croat. Chem. Acta 2007, 80, 187-191.

6. Bermudo, S.; Hernández-Gómez, J.C.; Sigarreta, J.M. On the total k-domination in graphs. Discuss. Math. Graph Theory 2018, 38, 301-317. [CrossRef] 
7. Cockayne, E.J.; Dawes, R.M.; Hedetniemi, S.T. Total domination in graphs. Networks 1980, 10, $211-219$. [CrossRef]

8. Henning, M.A.; Kazemi A.P. k-tuple total domination in graphs. Discret. Appl. Math. 2010, 158, $1006-1011$. [CrossRef]

9. Klobučar, A. Total domination numbers of Cartesian products. Math. Commun. 2004, 9, 35-44.

10. Klobučar, A. Domination numbers of cardinal products. Math. Slovaca 1999, 49, 387-402.

11. Gutman, I. Hexagonal systems, a Chemistry Motivated Excursion to Combinatorial Geometry. Teach. Math. 2007, 18, 1-10.

12. Majstorović, S.; Došlić, T.; Klobučar, A. k-Domination on hexagonal cactus chains. Kragujev. J. Math. 2012, 36, 335-347.

(C) 2019 by the authors. Licensee MDPI, Basel, Switzerland. This article is an open access article distributed under the terms and conditions of the Creative Commons Attribution (CC BY) license (http:/ / creativecommons.org/licenses/by/4.0/). 